\title{
Leptospirosis research: fast, easy and reliable enumeration of mobile leptospires
}

\author{
STEFAN SCHREIER ${ }^{1}$, WANNAPONG TRIAMPO ${ }^{2,3, *}$, GALAYANEE $^{2}$ \\ DOUNGCHAWEE $^{4}$, DARAPOND TRIAMPO ${ }^{2,3,5}$, and SUDARAT CHADSUTHI ${ }^{2,3}$
}

\footnotetext{
${ }^{1}$ Department of Bioengineering, Faculty of engineering physics, University of Applied Science Munich, 80335, Germany.

${ }^{2}$ Group of Biological and Environmental Physics, Department of Physics, Faculty of Science, Mahidol University, Bangkok 10400, Thailand.

${ }^{3}$ Center of Excellence for Vectors and Vector-Borne Diseases, Faculty of Science, Mahidol University, Salaya Campus, Nakhon Pathom 73170, Thailand.

${ }^{4}$ Department of Pathobiology, Faculty of Science, Mahidol University, Bangkok 10400, Thailand.

${ }^{5}$ Department of Chemistry (R3/1), Faculty of Science, Mahidol University, Salaya Campus, Nakhon Pathom 73170, Thailand.
}

\begin{abstract}
Leptospirosis caused by Leptospira interrogans is the most widespread zoonosis and a major public health problem worldwide. Based on light-scattering and absorption, quantification of leptospires using UV-VIS spectroscopy was used as an indirect counting technique by measuring the optical density and comparing this to automated direct counting using a counting chamber in combination with imaging and analyzing software. Two serovars, Bangkok and Copenhagenii, from log-phase growth were used for the establishment of standard curves. They were found to be linear and slightly different in gradient for each serovar. The ease, rapidity, and reliability of these two adapted and optimized counting techniques may provide a useful alternative enumeration technique for leptospirosis research.
\end{abstract}

Key terms: leptospirosis, leptospira, enumeration, spectroscopy, indirect counting.

\section{INTRODUCTION}

Leptospirosis is the most widespread zoonosis worldwide, particularly in warm and humid regions, caused by pathogenic spirochete of the genus Leptospira [1]. The disease is transmitted via indirect contact with contaminated water and soil [2] or direct exposure to infected animals and their products $[3,4]$, mainly urine. Both wild and domestic animals, such as rodents, heifers, and canines are the natural hosts. The estimated annual worldwide number of leptospirosis cases is 350,000-500,000 according to the World Health Organization's (WHO) - International Leptospirosis Society (ILS) survey [5]. The problem is more severe in humid tropical and subtropical countries such as Thailand [6], Nicaragua [7] and India [8].
Typically, leptospires are aerobic and motile spirochetes with helical or spiral structures and a unique (among the spirochetes) hook at both ends (Fig. 1). They are about 0.1-0.2 $\mu \mathrm{m}$ wide and 6-20 $\mu \mathrm{m}$ long and have a helical amplitude of approx 0.1$0.15 \mu \mathrm{m}$ and wavelength about $0.5 \mu \mathrm{m}[1,9]$. Their ultra-structure comprises a double cytoplasmic membrane, in close contact with a peptidoglycan layer and an outer membrane [10]. The composition of lipopolysaccharide (LPS) of their outer membrane is similar to other gram-negative bacteria [11], but has lower endotoxic activity [12]. Optimal conditions for the growth of this organism are well documented $[1,13]$. The most suitable conditions for their survival outside the host are a moist environment with a neutral $\mathrm{pH}$ and a temperature range between $20-32^{\circ} \mathrm{C}$. 


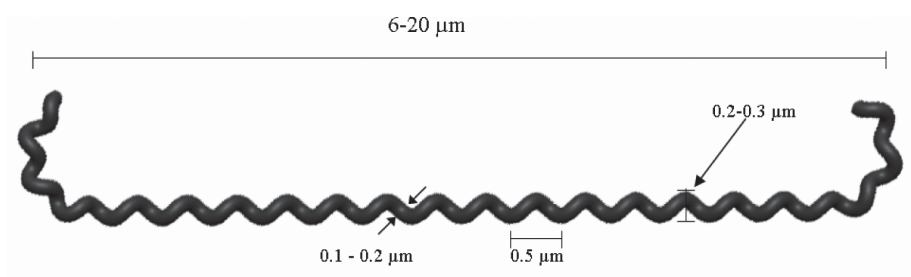

(a)

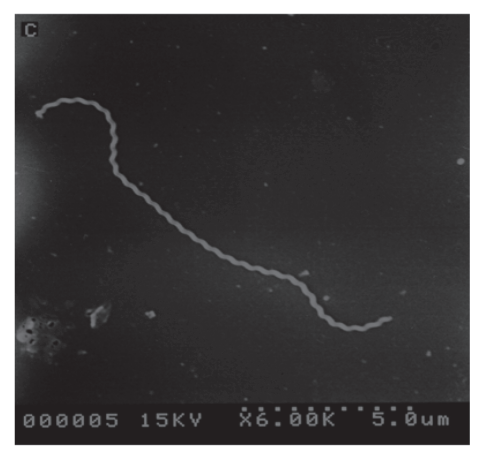

(b)

Figure 1: Pictures of a lepto spire: (a) side view schematic representation of the structure of Leptospira (b) SEM micrographs of spiral shape taken using scanning electron microscope (Hitachi, Japan) with $15 \mathrm{kV}$ [Magnification 6000x].

Leptospirosis research often requires the quantitative determination of bacterial populations. Enumerating microbial populations is also important for evaluating such products as antibiotics, vitamins, and preservatives. Quantification is also necessary to prepare inocula for bioassays and tests. There are numerous well-known direct, as well as indirect methods, for enumerating bacteria, for instance counting by means of a counting chamber, plate counting, filtration, optical or turbidimetric procedures, and the Coulter counter [14]. For leptospires, the well-accepted method of enumerating bacteria, which remains the gold standard, is direct counting the spirochetes using a direct counting chamber and dark-field microscopy [2]. However, there are three main features that handicap the execution and might distort the outcome of the counting experiment. These are movement, the low refractive index, which is equal to water, and the necessity to breed the cells in more or less a liquid media. If not prevented, the movement of leptospires causes clumping into cultures, so that the colonies cannot be traced back to one single cell [1]. Other problems include regulation of humidity and oxygenation. The plate should not dry out and too much oxygen inhibits the growth of the leptospires. As well, the largely disadvantageous incubation period of 10 to 20 days prevents fast quantification of samples. Because leptospires are less visible under a dark field microscope with a counting chamber slide than with a routine glass slide, the movement of the bacteria makes manual counting rather difficult. Counting only works with the assumption of random 
movement, with a migration in all directions, so that the organisms on two adjacent sides of the squares of the counting grid and within the squares are being counted. However, moving objects require far more concentration from the reader and errors are presumably not negligible. Additionally, the samples have to be diluted properly to obtain the amount appreciable to the human eye for counting, depending on the initial cell concentration. There is doubt whether this method guaranties reproducibility. Recently, enumeration with a Coulter counter appears to be a feasible and sufficiently precise alternative for the quantification of a concentration [15]. However, a device specially adapted to the counting of leptospires is needed, which makes this method rather expensive.

In this work, optical methods for indirect counting of leptospires include the measurement of turbidity and optical density with a spectrophotometer. Measurement of turbidity is seldom used due to its low sensitivity. However, measurement of optical density seems to be very promising and is an already proven method with other bacteria, which is fast, easy to handle, inexpensive, and less time consuming [16]. It has been reported that DNA absorbs light at the wavelength of 260 $\mathrm{nm}$ [17, 18]. In an earlier work with leptospirochetes, optical measurements at a wavelength of $400 \mathrm{~nm}$ have been shown to yield the highest resolution for undiluted culture media. Here, two already existing methods for enumeration have been adapted and optimized. These are direct counting in combination with image processing and analyzing software, called automated direct counting (ADC) and indirect optical density (OD) based counting using UV-VIS spectroscopy. Standard curves were established in order to determine whether firstly, linearity between these two techniques could be established for a standard curve over different concentrations, and secondly, in the case of linearity, whether there is a difference between the two serovars in evidence, regarding the individual standard curves. The main idea behind software supported counting, in addition to the advantages of offering a very fast and simple means of execution, is the reproducibility of counting errors, contrary to operator error, which may have considerable variation. In our view, the ease and rapidity of counting with UV-VIS spectroscopy makes this technique superior to the direct method. However, we would like to note that we have applied this method only to measure two different serovars. Since comparable studies are made with more leptospira serovars, it is perhaps not wise to assume that these proposed protocols would work with the different strains and media used for growth conditions.

\section{METHODOLOGY}

Pathogenic Leptospira interrogans serovars Bangkok and Copenhagenii were originally obtained from the National Leptospirosis Reference Center, the National Institute of Health (NIH), Thailand. Stock cultures were grown in Ellinghausen and McCullough liquid medium as modified by Johnson and Harris (EMJH) [1] in $27^{\circ} \mathrm{C}$ incubator. These cultures were subcultured for concurrent weekly use and mediumterm maintenance.

\section{Direct Counting}

Direct counting was performed by using a counting chamber and dark field microscopy. A 1:100 dilution of inoculum cultures were prepared using PBS-buffer $1: 3$ in order to adjust the cell amount to be within a countable range. $10 \mu \mathrm{l}$ of diluted inoculum samples were then loaded individually into the counting chamber. During the enumerating process, pictures were taken with an Olympus DP70 CCD camera equipped with an Olympus BX60 microscope with a dark field condenser at a magnification of $100 \mathrm{x}$ and a resolution of 1360 x 1024 pixels. Digital image analysis was performed with Image Pro Version 5.1 software. Limited by the size of the CCD camera chip, the area of interest for each image was $750 \mu \mathrm{m}$ x $500 \mu \mathrm{m}$. Only six squares of the counting chamber per picture 
within the frame (Fig. 2) could be taken for subsequent analysis. A total of twelve squares were used for analysis with a total size of $0.75 \mathrm{~mm} \times 1 \mathrm{~mm}$. For each of the twelve squares, the image of the fourth focused layer was captured for analysis (Fig. 2). While the counting of eight focused layers was found to reflect approximately the real number of cells counted in the culture media, it was found that the sum of the cells is more or less in the multiple of 7 for the fourth focused layer (data not shown). Taking pictures at the very fourth focused layer will not only save time and disk space, but also a better quality of the image can be obtained for processing, due to greater distance to bottom and cover slide, thus increasing the signal-to-noise ratio. This fourth layer is obtained by locating the exact focus of the bottom of the counting slide and sliding up the stage of the microscope by $50 \mu \mathrm{m}$. Obtained values were averaged to minimize data variation. It is important to remark that purely manual counting would necessitate the reading of a total number of eight pictures consisting of at least a hundred bacteria per picture. Such manual counting can result in considerable human error. The use of processing software, Image Pro, allows automatic enumeration of the bacteria from an image. Prior to using the macro for cell counting, the image was first converted to a 16-bit greyscale. HiGauss filtering was then performed to enhance the fine details. Each ADC count was performed with a freshly cleaned and loaded chamber slide.
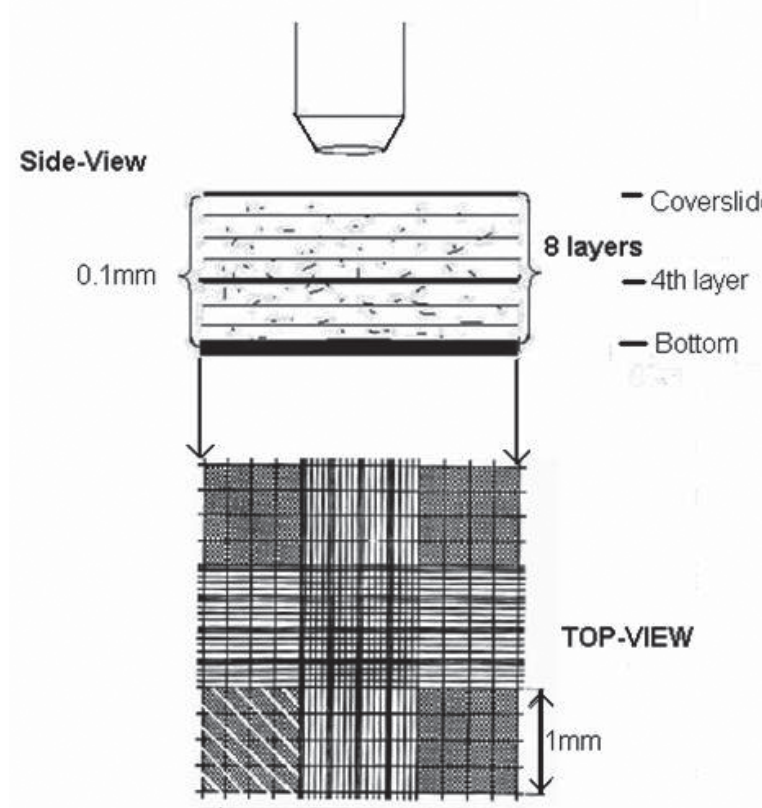
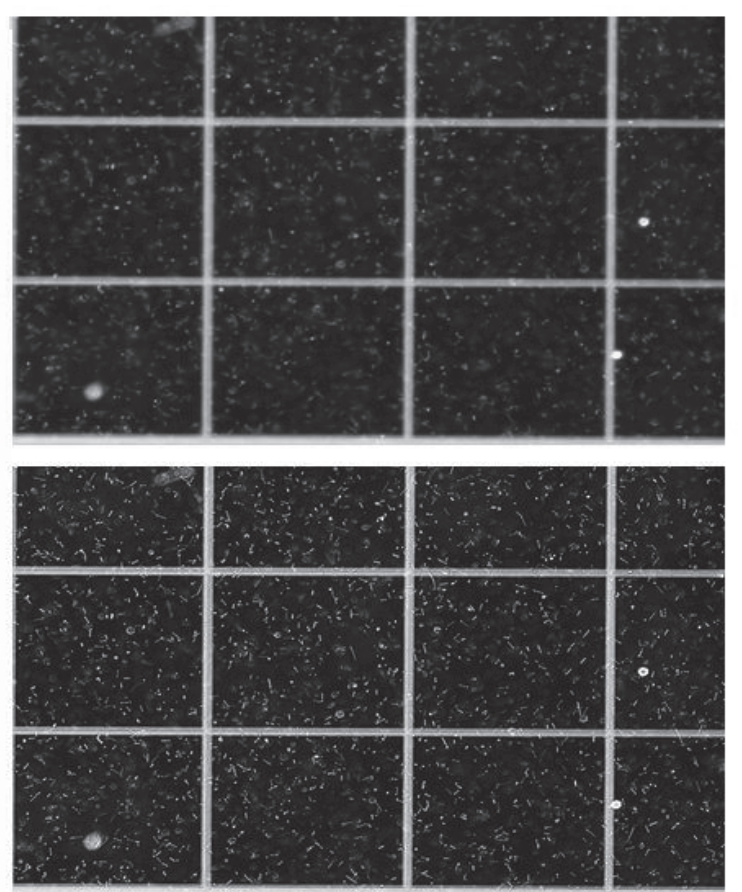

Figure 2: Principle of the focused layers and the counting grid. Each line in the side view represents a focused layer, where leptospires are best visible. The thick line indicates the middle layer. The top view illustrates the grid of the counting chamber slide. The size of the marked area (diagonal stripes) amounts $1 \mathrm{~mm} \leq$ and contains 16 squares. Only 12 non-overlapping squares were used for picture analysis. 


\section{Indirect Counting}

Indirect counting using UV-VIS spectroscopy (V-530 UV/VIS spectrometer, code number 6736-J004A, Jasco International Co., Ltd.) was performed using five tubes for each strain. Measurements were obtained using a quartz cuvette (SUPRASIL 200- $2500 \mathrm{~nm}$, light path $10 \mathrm{~mm}$, type number 100.600-QG, Hellma Co.) prior to OD measurement of the real sample, a baseline of the solution was first measured. The baseline solution contained no leptospires and was prepared in the same way as the sample solution. The spectrum ranging from 230 - $450 \mathrm{~nm}$ was recorded. The wavelengths of interest were at 260 and $400 \mathrm{~nm}$. The absorbance was calculated following the Lambert-Beer law [19]. The sample was firstly centrifuged for at least 30 minutes at $14000 \mathrm{rpm}$. The supernatant of the process was used as baseline solution. The dilution of the EMJH-media with phosphate buffered saline (PBS) 1:7 was necessary only for OD measurements at the wavelength of $260 \mathrm{~nm}$. Before centrifugation, $0.5 \mathrm{ml}$ of PBS was added to each microtube as a pre-dilution step of the media. After centrifugation, 1.0 $\mathrm{ml}$ from the culture was carefully obtained and used as the initial baseline solution. After that, the packed cells are completely resuspended in the remaining $0.5 \mathrm{ml}$ of culture media. $220 \mu \mathrm{l}$ was then taken and diluted with $1 \mathrm{ml}$ of PBS. The exact same procedure was repeated for the baseline solution. At $400 \mathrm{~nm}$, it is possible to work with the pure media for baseline correction of the OD measurement and the sample itself. As there were also proteins in the media but no nuclei acid, the measurement at $260 \mathrm{~nm}$ is far more sensitive to determine low cell concentration. The experiments were aborted after the OD reached the highest value of 0.6 absolute value, due to significant non-linearity beyond this threshold.

\section{RESULTS AND DISCUSSION}

The measurements were made with the aim of demonstrating the ease, rapidity, and reliability of the proposed optimized counting techniques that may provide useful alternatives for enumeration in leptospirosis research. Fig. 3 shows the optical density recorded as a function of time for the two serovar cultures, namely Bangkok and Copenhagenii. The scattering and absorption depends on optical properties of the colloidal particles and the solution, including the refractive index and the size of the particles, the refractive index of the solution, the wavelength and the intensity of the light source. With the appropriate setup and prior knowledge of the solution, the properties of the scattering agents, such as the size or the concentration, can be deducted. When illuminating a scattering agent, the scatter pattern can be expressed by $I=I_{0} P(\theta)$ where $I_{0}$ is the input intensity and is the angular distribution of scattered light as a function of the deflection angle. Although can be solved analytically, its approximation function, such as HenyeyGreenstein function [22],

$$
P(\theta)=\frac{1}{4 \pi} \frac{1-\mathrm{g}^{2}}{\left(1+\mathrm{g}^{2}-2 \mathrm{~g} \cos \theta\right)^{3 / 2}}
$$

has been used successfully for computation modelling [23], where is the average of the cosine of the scattering angle. It was found that the growth curves (Gompertz growth function) for these two serovars differ very slightly from each other. This measured optical density follows the expected behaviour reported in the literature [20]. As mentioned before, measurement at $260 \mathrm{~nm}$ was used because it correctly reflects the total nucleotide contents [21].

Fig. 4 shows the linearity of the optical density versus automated count plot obtained only from points of the exponential growth phase of the experiments. For the settings of these experiments, the exponential phase ends mainly before the OD of 0.6 absolute value has been reached. There are two fitting curves visible. The brighter one is calculated only from the 6 points of the serovar Copenhagenii and so is the darker one for the serovar Bangkok. All points are 


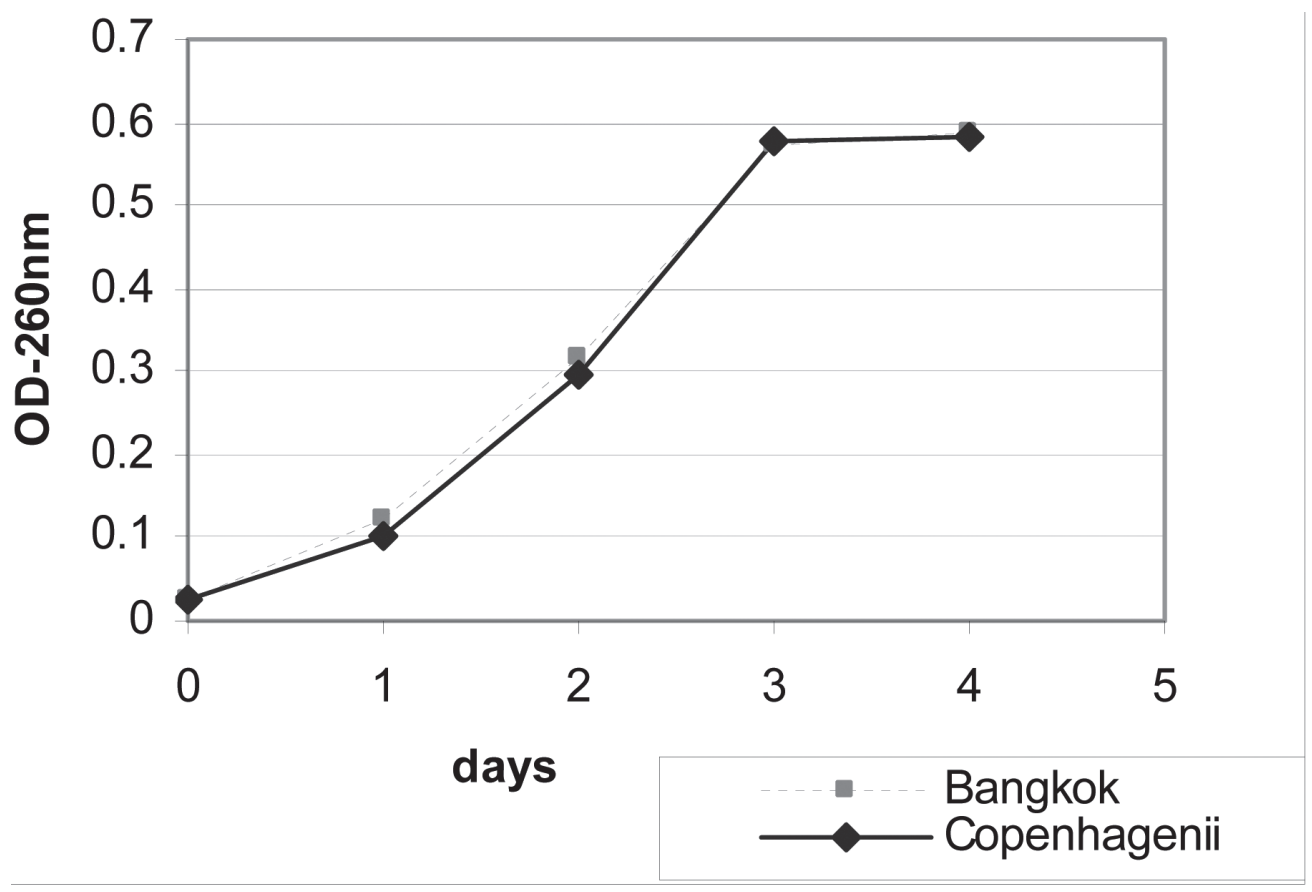

Figure 3: Growth curves measured with optical density spectrometry at $260 \mathrm{~nm}$ for the serovar (a) Bangkok and (b) Copenhagenii.

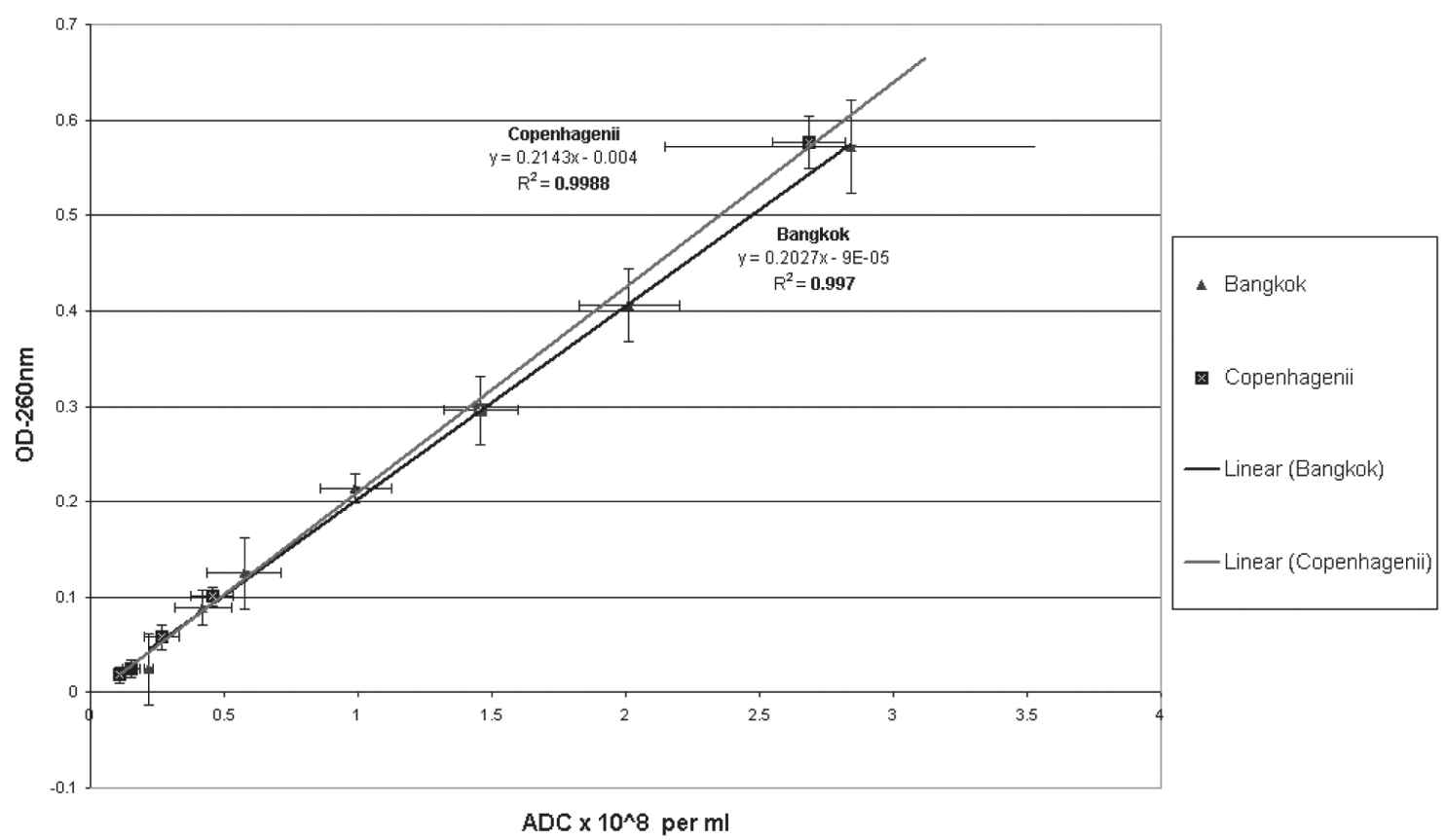

Figure 4: Graph of optical density versus automated direct counting. The two fitting curves have been constructed by Microsoft Excel and indicate two standard curves for the serovars Copenhagenii and Bangkok. 
derived from five independent samples with one left out, so that the twelve points in the chart are the average of four measurements. The averaged points fit to a high degree $(\mathrm{R} \leq=0.998)$ to the fitting curves, so that linearity can be assumed. The results for the stationary phase are especially consistent with former works [14], where the optical characteristics of Escherichia coli have been explored. Limitations of this study include the lack of evaluation of this technique on different types of cultures or directly from infected tissues or fluids. Future work should include a more extensive evaluation of the UV-VIS for leptospirosis enumeration, to include from other sources such as tissue culture and directly from experimentally infected animals. For precise measurements with UV-VIS spectrometry, it is necessary to gather the background spectrum before the actual measurement of the real sample to avoid manual inaccuracy. However, the cells in solution need to be washed completely from the media, which is inapplicable for the leptospire cells. Due to their marginal weight, it is necessary to centrifuge them at least for 30 minutes at $14000 \mathrm{rpm}$. The total cell-washing step would take at least two hours. A faster method would be to dilute the media to the necessary limit for the device and measure the baselines of each sample gained during preparation of the solutions. A disadvantage however, is the resolution limit for concentrations lower than $10^{7}$. Centrifugation in the matter of counting cells should be regarded with care as cells may be lost or destroyed. Counting with a counting chamber in contrast does not need a centrifugation step at all and enables the quantification of leptospira cells at the concentrations ranging from $10^{5}$ to $10^{8}$.

In summary, leptospirosis research is complicated by the lack of an efficient technique for quantification. This study demonstrates the use of UV-VIS spectroscopy as an indirect counting method and automated direct counting method as alternatives to determine number of the leptospire. Limitations of this study include the lack of evaluation of these techniques on different types of cultures or directly from infected tissues or fluids. Future work should include a more extensive evaluation of both techniques for leptospira enumeration, to include from other sources such as directly from experimentally infected tissues or using various kinds of serovars.

\section{ACKNOWLEDGMENTS}

This work has been supported in part by the Thailand Center of Excellence in Physics, the Thailand Research Fund (TRF), the Commission on Higher Education (CHE), the National Center for Engineering and Biotechnology (BIOTEC) Thailand, the Academy of Sciences for the Developing World (TWAS), and the University of Applied Science, Munich.

\section{REFERENCES}

[1] FAINE S, ADLER B, BOLIN C, PEROLAT P (1999)

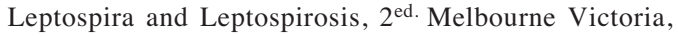
Australia: MediSci: 173-174

[2] LEVETT PN (2001) Leptospirosis. Clin Microbiol Rev 14: $296-326$

[3] TANGKANAKUL W, THARMAPHORNPIL P, PLIKAYTIS BD, BRAGG S, POONSUKSOMBAT D, CHOOMKASIEN P, KINGNATE D, ASHFORD DA (2000) Risk factors associated with leptospirosis in northeastern Thailand, 1998. Am J Trop Med Hyg 63: 204-208

[4] DOUGLIN CP, JORDAN C, ROCK R, HURLEY A, LEVETT PN (1997) Risk factors for severe leptospirosis in the parish of St. Andrew, Barbados. Emerg Infect Dis 3: 78-80

[5] INTERNATIONAL LEPTOSPIROSIS SOCIETY (ILS). In: Proccedings of the fourth ILS 2005 abstracts, Chiang Mai, Thailand, November 14-16, 2005

[6] WATT G, JONGSAKUL K, SUTTINONT C (2003) Possible scrub typhus coinfections in Thai agricultural workers hospitalized with leptospirosis. Am J Trop Med Hyg 68: 89-91

[7] BRANDLING-BENNETT AD, PINHEIRO F (1996) Infectious diseases in Latin America and the Caribbean: are they really emerging and increasing. Emerg Infect Dis 2: 59-61

[8] World Health Organization. Leptospirosis, India: Report of the investigation of a post-cyclone outbreak in Orissa, Wkly Epidemiol Rec WHO, Nov. 1999, pp. 217-223

[9] GOLDSTEIN SF, CHARON NW (1990) Multipleexposure photographic analysis of a motile spirochete. Proc Natl Acad Sci (USA) 87: 4895-4899

[10] HAAKE DA (2000) Spirochaetal lipoproteins and pathogenesis. Microbiology 146: 1491-1504

[11] VINH T, ADLER B, FAINE S (1986) Ultrastructure and chemical composition of lipopolysaccharide extracted from Leptospira interrogans serovar Copenhagenii. J Gen Microbiol 132: 103-109 
[12] SHIMIZU T, MATSUSAKA E, TAKAYANAGI K, MASUZAWA T, IWAMOTO Y, MORITA $\mathrm{T}$, MIFUCHI I, YANAGIHARA Y (1987) Biological activities of lipopolysaccharide-like substance (LLS) extracted from Leptospira interrogans serovar Canicola strain Moulton. Microbiol Immunol 31: 727-735

[13] FAINE S. Guideline for control of leptospirosis, World Health Organization, Geneva 1982, Vol. 67, pp. 129

[14] PHILIPP G, KRIEG NR, MURRAY RGE, WOOD WA (1994) Methods for general and molecular bacteriology. American Society for Microbiology: 261266

[15] HUMBERD CM, MURRAY CK, STUART SK, REEB BA (2005) Enumerating leptospires using the Coulter Counter. Am J Trop Med Hyg 73: 962-963

[16] ALUPOAEI CE, GARCIAUBIO LH (2004) Growth Behavior of Microorganisms using UV-VIS spectroscopy: Escherichia coli. Biotechnol Bioeng 86: 163-167

[17] FREIFELDER D (1982) Physical Biochemistry, Applications to Biochemistry and Molecular Biology, $2^{\text {nd }}$ ed, WH Freeman and Company, New York: 504
[18] INAGAKI T, HAMM RN, ARAKAWA ET, PAINTER LR (1997) Optical and dielectric properties of DNA in the extreme ultraviolet. J Chem Phys 61: 4246- 4250

[19] PERKAMPUS HH (1992) UV-VIS spectroscopy and its application. New York: Springer-Verlag Berlin Heidelberg

[20] WONG-EKKABUT J (2007) Effects of electromagnetic fields on biological systems : Leptospira. Thesis (Ph.D. (Physics)) Mahidol University, CALL NUMBER: Thesis J61e 2007 (Reserve)

[21] FREIFELDER D (1982) In: Physical biochemistry, 2nd ed. San Francisco: W.H. Freeman \& Company: 504

[22] HENYEY LG, GREENSTEIN JL (1941) Diffuse radiation in the galaxy. Astrophys J 193: 70-83

[23] MOURANT JR, FREYER JP, HIELSCHER AH, EICK AA, SHEN D, JOHNSON TM (1998) Mechanisms of light scattering from biological cells relevant to noninvasive optical-tissue diagnostics. Applied Optics 37: $3586-3593$ 